\title{
DESIGN OF WIRELESS DEVICE TO MEASURE PLANTAR PRESSURE AND GAIT ANALYSIS
}

\author{
Mahendra Khatri, Sambardhan Dabadi, Sandeep K. Shrestha, Saugat \\ Acharya, Sudip Tamang, Swornima Shakya
}

\begin{abstract}
Foot plantar pressure is the pressure field that acts between the plantar region of the foot and supporting ground. The pressure exerted on the variable region of the foot can be determined using discrete pressure sensors. Information obtained from these sensors is useful in the measurement of gait and posture for diagnosing various problems associated with a lower limb, footwear design, and sports biomechanics.
\end{abstract}

This project is aimed to design a portable in-shoe plantar pressure and gyroscopebased gait angle measurement system. Six Force Sensitive Resistor (FSR) placed in the sole (hallux, 1st, 5th metatarsal, midfoot lateral, midfoot medial and heel respectively) detects the plantar pressure and gyroscope placed at the ankle, knee and hip help measure the orientation and angle of joint movement during various phases of gait. The study among $\mathbf{1 6}$ male and $\mathbf{1 6}$ female subjects illustrated the significant pressure variation $(\mathrm{p}<0.0001, \mathrm{t}=5.17$ with $\alpha=95 \%)$. Similarly, there was a significant difference in pressure between normal and fast walking speed $(p<0.0001, t=5.88)$ with mean values of $353 \mathrm{Kpa}$ and $426 \mathrm{Kpa}$ respectively. The mean pressure value for slow walking speed was 423Kpa while there was no significant variation between slow and normal walking speeds $(p=0.62, t=1.98)$. Plantar pressure increased linearly with an increase in the body weight of a person as well. The mean pressure for the 45-50 age group was $313.25 \mathrm{Kpa}$ and that for $70-75$ was $449 \mathrm{Kpa}$. The study among 10 diabetics and 10 non-diabetic subjects illustrated significantly higher pressure on 1st and 5th metatarsal on diabetic subjects $(p=0.0207$ and $t=2.536)$.

The movement of ankle, knee and hip joint is visualized using the 3D model of a lower limb through processing software. The study illustrated the range of ankle joint movement between -60(dorsiflexion) to 200 (plantarflexion), for knee joint was 00 to 300 (flexion) and that for hip joint was -50 (extension) to 400 (flexion). There was a significant difference in angular values for all three joints while climbing up and down the staircase as compared to walk in a level surface.

Keywords: Foot plantar pressure, force sensitive resistor, gyroscope, gait analysis, joint angle, wireless system.

\section{INTRODUCTION}

Foot, the most important biomechanical structure provides support and stability to the whole body during everyday activities(1). Foot plantar pressure is the pressure field that acts between the plantar region of the foot and supporting ground(2). The information of foot plantar pressure helps the physicians to diagnose various foot-related problems and is useful in footwear designing. This is also helpful in the analysis of the gait cycle 
and obtained deviated pressure values indicate the problems associated with gait(3). Excess pressure on any region of the foot can lead to neuromuscular diseases and be the cause of ulceration of foot, which is mostly observed in the people with type II diabetes (4). It is estimated that approximately 300million people globally are suffering from diabetic foot neuropathy and foot related ulceration in the year 2011 which is predicted to rise to 438 million by 2030 . Plantar pressure varies on different factors. Some of these include foot-type, walking speed, gender, age, weight, body mass index and so on $(5,9,10,11,12,13)$. Besides these, some disease conditions including parkinsonism and diabetes have shown a greater impact on plantar pressure $(6,7)$.

This project focuses on the development of an in-shoe system that can monitor the pressure exerted on a different region of the foot during various phases of gait without any disturbance to the walking pattern of the user. Additionally, the sensors placed on ankle, knee, and hip provides the information of joint angle during different gait phases and help detect any deviation in normal gait pattern.

Present available quantitative evaluation of plantar pressure and gait is limited by the availability, size, and cost(9). The assessment is achievable only in hospitals of urban areas. While in rural areas plantar pressure and gait analysis are carried out by the manual observational procedure. These procedures are inefficient and inaccurate to determine any deviation in plantar pressure and gait. So, there is a need for a portable, accurate and efficient as well as an economical device for the measurement of foot plantar pressure and gait analysis.

\section{Material Required}

\subsection{Hardware}

\subsubsection{Force Sensitive Resistors (FSR)}

Force sensitive resistors sensors are basically a resistor that changes its value depending on how much it is pressed. FSRs electrical resistance decreases as an increase in load weight or pressure. They are low cost and easy to use sensors. To assess pressure distribution FSRS is selected to be placed underneath the sole.

\subsubsection{GY521 MPU-6050}

The MPU-6050 is a 6DOF (Degrees of Freedom) or a six-axis IMU sensor, which mean that it gives six values as output i.e. three values from the accelerometer and three from the gyroscope. The MPU-6050 is a sensor based on MEMS (Micro Electro Mechanical Systems) technology. The MPU-6050 sensor contains MEMS accelerometer and MEMS gyro in a single chip. It is very accurate, as it contains 16-bits analog to digital conversion hardware for each channel. Therefore, it captures the $\mathrm{x}, \mathrm{y}$, and $\mathrm{z}$ channel at the same time.

\subsubsection{Arduino Mega (ATmega2560)}

The Arduino Mega is a microcontroller board based on the ATmega1280. It has 54 digital input/output pins (of which 14 can be used as PWM outputs), 16 analog inputs, 4 UARTs 
(hardware serial ports), a $16 \mathrm{MHz}$ crystal oscillator, a USB connection, a power jack, an ICSP header, and a reset button. It contains everything needed to support the microcontroller.

\subsubsection{Bluetooth Module (HC-05)}

The HC-05 is a module which can add two-way (full-duplex) wireless functionality. It can be used to communicate between two microcontrollers like Arduino or communicate with any device with Bluetooth functionality like a Phone or Laptop. The module communicates with the help of USART at 9600 baud-rate hence it is easy to interface with any microcontroller that supports USART.

\subsection{Software}

\subsubsection{Processing IDE}

Processing is a flexible software sketchbook and a language for learning how to code within the context of the visual arts. Since 2001, Processing has promoted software literacy within the visual arts and visual literacy within technology. There are tens of thousands of students, artists, designers, researchers, and hobbyists who use Processing for learning and prototyping.

\subsubsection{Arduino IDE}

The Arduino integrated development environment (IDE) is a cross-platform application that is written in the programming language Java. It is used to write and upload programs to the Arduino board. The source code for the IDE is released under the GNU General Public License, version 2. The Arduino IDE supports the languages $\mathrm{C}$ and $\mathrm{C}++$ using special rules of code structuring. The Arduino IDE supplies a software library from the Wiring project, which provides many common input and output procedures. The userwritten code only requires two basic functions, for starting the sketch and the main program loop, that is compiled and linked with a program stub main() into an executable cyclic executive program with the GNU toolchain, also included with the IDE distribution.

3. Result

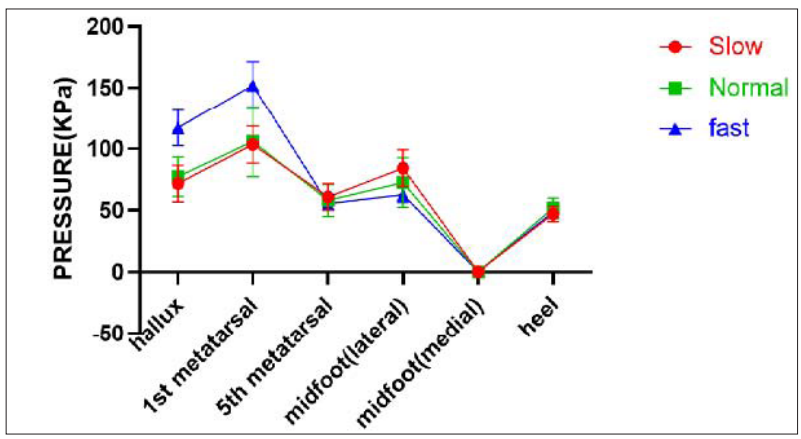

Figure 1: Pressure variation according to walking speed 


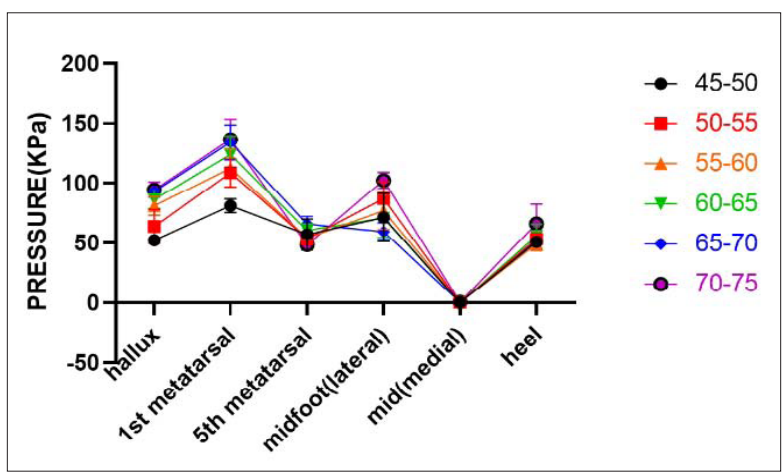

Figure 2: Pressure Variation according to body weight

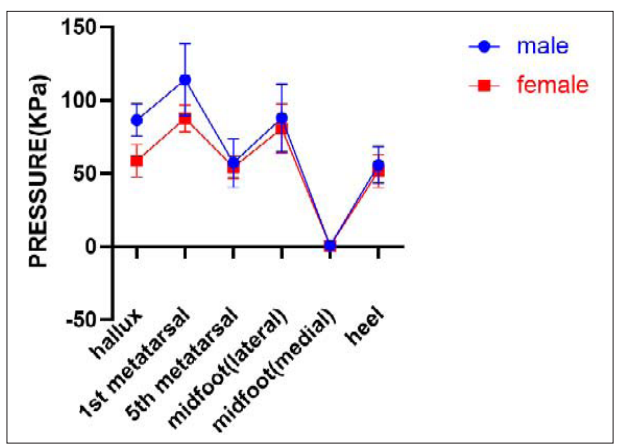

Figure 3: Pressure Variation according to gender

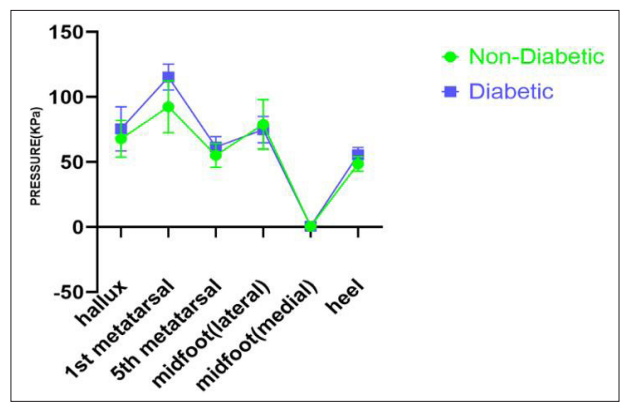

Figure 4: Pressure Variation between diabetic and non-diabetic subjects

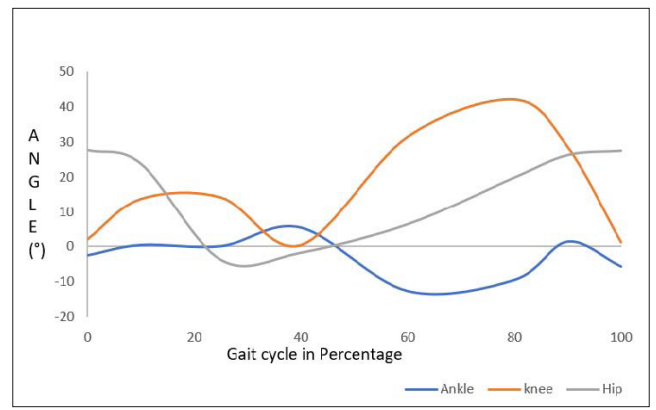

Figure 5: Joint angle at different gait phas

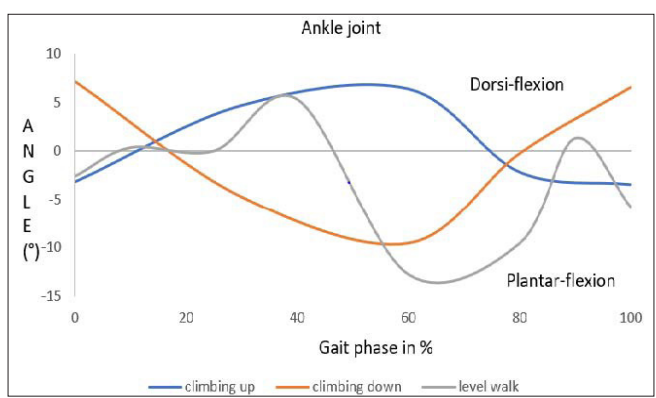

Figure 6: Ankle Joint Angle Variation While Climbing Staircase Up and Down 


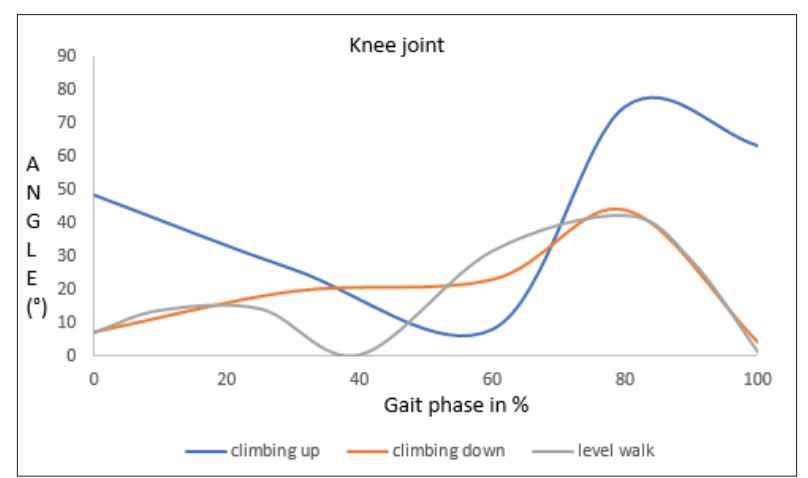

Figure 7: Knee Joint Angle Variation While Climbing Staircase Up and Down

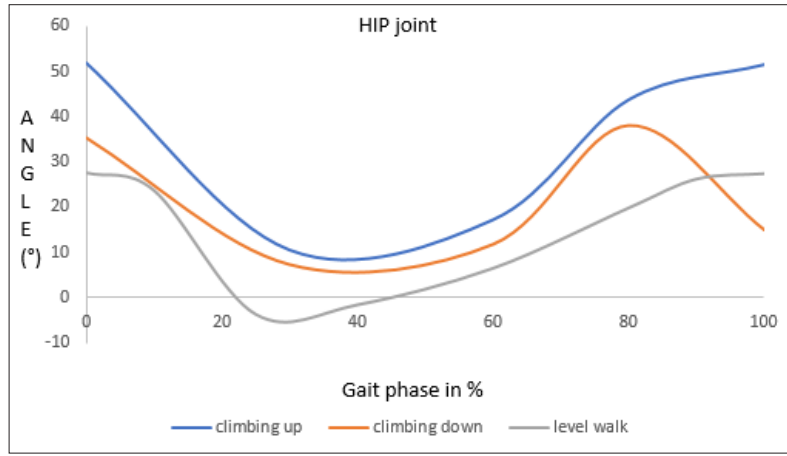

Figure 8: Hip Joint Angle Variation While Climbing Staircase Up and Down

Data collected from various subjects were classified based on the walking speed, body weight, gender and diabetic and non-diabetic. Figure 5 represents the variation of plantar pressure with walking speed. It is observed that pressure increased when the walking speed deviated from normal walking speed $(0.96 \mathrm{~m} / \mathrm{s}$ and stride length $0.52 \mathrm{~m})$. Figure 6 illustrates the change in pressure with an increase in body weight. The pressure increase is linear with increase in body weight. Similarly, from figure 7 it is observed that male experience higher pressure on hallux and 1st metatarsal than female while the variation is not significant in rear-foot. Figure 8 compares the pressure variation between diabetic and non-diabetic subjects. The pressure is quite higher in 1st metatarsal for the diabetic subject while there is no significant variation on another region of the foot.

Figure 9 represents the joint angle change during various phases of the gait cycle. From the graph the range of motion for ankle joint was 60 plantar flexion to 230 dorsiflexions, knee joint was 00 to 450 flexion and for hip joint was 40 extension to 300 flexions.

Figure 10,11,12 represents the comparison of the change in angle of the ankle, knee, and hip joint during climbing the staircase up and down respectively. It is observed that the ankle joint is mostly dorsiflexed while climbing up and is mostly plantar flexed while climbing down. The knee is flexed to about 800 while climbing up while the flexion is limited to 550 while climbing down. However, the pattern of angular changes for hip joint 
was quite similar for climbing up and down the stairs.

\section{Discussion}

The results were compared based on different factors that affected the plantar pressure. Basically, the subjects were classified according to gender, body weight, and walking speed. Study on 16 male and 16 female suggested male experience higher plantar pressure on hallux and 1st metatarsal than female, which is because of the greater weight and walking speed of male. This difference was significant with $\mathrm{p}<0.0001$ and $\mathrm{t}=5.17$. On other study 10 subjects were asked to walk slow $(0.56 \mathrm{~m} / \mathrm{s})$, normal $(0.96 \mathrm{~m} / \mathrm{s})$ and fast $(1.4 \mathrm{~m} / \mathrm{s})$. This showed that the plantar pressure increases while the walking speed is increased as well as decreased. On increasing the walking speed, the impact force was higher that caused higher pressure on the foot. While decreasing the speed, contact time increased leading to greater plantar pressure. However, the difference was not significant for a slow and normal walk as suggested by t-test with $\mathrm{p}=0.629$ and $\mathrm{t}=1.98$. The variation of plantar pressure with the change in body weight was studied among 30 subjects of various weights. The subjects were classified into six groups with five in each group. The data illustrated that the pressure increases linearly with an increase in body weight. There was no significant difference in any region foot. The average pressure experienced by people of age group $45-50 \mathrm{~kg}, 50-55 \mathrm{~kg}, 55-60 \mathrm{~kg}, 60-65 \mathrm{~kg}, 65-70 \mathrm{~kg}$ and $70-75 \mathrm{~kg}$ were $313 \mathrm{Kpa}, 367 \mathrm{Kpa}, 374 \mathrm{Kpa}, 397 \mathrm{Kpa}, 405 \mathrm{Kpa}$, and $448 \mathrm{Kpa}$ respectively. This increase in pressure with body weight is due to a higher load in the foot.

The next study on 10 diabetic patients with an average weight of $66.8 \mathrm{~kg}$ and the average age of 44.3 years visualized the significant difference in plantar pressure as compared to normal subjects. The results showed higher pressure on 1st and 5th metatarsal, with pressure values $115.24 \mathrm{Kpa}$ and $61.5 \mathrm{Kpa}$ respectively. The total pressure on foot for diabetic subjects was $383 \mathrm{Kpa}$ while that for non-diabetic subjects was $343 \mathrm{Kpa}$. There is a significant difference in pressure as suggested by t-test with a $p$-value of $0.0207(p<0.05)$ and $t$ value of 2.536. The increase in pressure is due to the greater weight of diabetic subject as compared to non-diabetic subjects and due to their health condition. ${ }^{1}$

1. Chan LK. 1 - The anatomy of the human foot. Handb Footwear Des Manuf. 2013;3-26.

2. Motha L, Kim J, Kim WS. Instrumented rubber insole for plantar pressure sensing. Org Electron [Internet]. Elsevier B.V.; 2015;23:82-6. Available from: http://dx.doi.org/10.1016/j. orgel.2015.04.020

3. Varma M. Arduino-Based Foot Neuropathy Analyzer.

4. Abdul Razak AH, Zayegh A, Begg RK, Wahab Y. Foot plantar pressure measurement system: A review. Sensors (Switzerland). 2012;12(7):9884-912.

5. Okuno R, Fujimoto S, Akazawa J, Yokoe M, Sakoda S, Akazawa K. Analysis of spatial-temporal plantar pressure pattern during gait in Parkinson's disease. Conf Proc IEEE Eng Med Biol Soc. 2008;2008:1765-

6. Deepashini H, Omar B, Paungmali A, Amaramalar N, Ohnmar H, Leonard J. An insight into the plantar pressure distribution of the foot in clinical practice: Narrative review. Polish Ann Med [Internet]. Polish Pediatric Society.; 2014;21(1):51-6. Available from: http://dx.doi. org/10.1016/j.poamed.2014.03.003

7. Drerup B, Szczepaniak A, Wetz. HH. Plantar pressure reduction in step-to gait: A biomechanical investigation and clinical feasibility study. Clin Biomech. 2008;23(8):1073-9.

8. Claverie L, Ille A, Moretto P. Discrete sensors distribution for accurate plantar pressure analyses. Med Eng Phys [Internet]. Elsevier Ltd; 2016;38(12):1489-94. Available from: http://dx.doi. 
Angular changes in joint angle were first visualized using the 3D model of the lower limb and then numerical values were stored in an excel file which was used for the further analysis. The joint angle changes from 10 subjects during normal walking speed $(0.96 \mathrm{~m} / \mathrm{s}$ and stride length of $0.52 \mathrm{~m}$ ) on a level surface was studied. The angular changes during each phase of gait were analyzed. For ankle joint, the range of joint movement was found to be -60 (dorsiflexion) to 200 (plantar-flexion). The peak angular deviation of 22.80 was observed during the pre-swing phase. For the knee joint, the range of joint motion was 00 to 300 . The peak angular change was 320 during the pre-swing phase. The range of motion for hip joint was determined between -50(extension) to 300(flexion) with a peak value of 27.20 during mid-swing phase. The angular variation during various activities was also studied, for this the subjects were asked to climb up and down the staircase. The data illustrated that the angular changes during climbing up the stairs and down the stairs is significantly different than in normal level surface walking. As compared to phases in level surface walk the phases in climbing the stairs are different. The peak value of the angular change in ankle joint was 100 during forwarding continuation, while that for knee joint was 500 during weight acceptance and that for hip joint was 550 during foot placement phase. This difference in angular changes during climbing is due to a greater level of the lifting of foot and lower extremities.

\section{Conclusion}

Many people experience uneven foot plantar pressure distribution on their foot; due to this reason, people face problems like ulceration (diabetic patient), neuromuscular disease, sprain, and even gait abnormalities. Uneven foot plantar pressure distribution, if not diagnosed earlier can cause ulceration which can also lead to amputation. Also, uneven foot plantar pressure distribution causes gait abnormalities which directly impact the player performance.

We have designed the device that could analyze the foot plantar pressure and gait. The device consists of FSR placed on the sole of the shoe. The pressure on each FSR causes the resistance of FSR to vary which is converted in terms of pressure. With the help of gyroscope, we analyzed the angular position of joints of lower limb which is used to

org/10.1016/j.medengphy.2016.09.021

9. Periyasamy R, Mishra A, Anand S, Ammini AC. Preliminary investigation of foot pressure distribution variation in men and women adults while standing. Foot [Internet]. Elsevier Ltd; 2011;21(3):142-8. Available from: http://dx.doi.org/10.1016/j.foot.2011.03.001

10. Putti AB, Arnold GP, Abboud RJ. Foot pressure differences in men and women. Foot Ankle Surg. 2010;16(1):21-4.

11. Machado ÁS, Bombach GD, Duysens J, Carpes FP. Differences in foot sensitivity and plantar pressure between young adults and elderly. Arch Gerontol Geriatr [Internet]. Elsevier Ireland Ltd; 2016;63:67-71. Available from: http://dx.doi.org/10.1016/j.archger.2015.11.005

12. Bosch K, Nagel A, Weigend L, Rosenbaum D. From "first" to "last" steps in life-Pressure patterns of three generations. Clin Biomech [Internet]. Elsevier Ltd; 2009;24(8):676-81. Available from: http://dx.doi.org/10.1016/j.clinbiomech.2009.06.001

13. Birtane M, Tuna $H$. The evaluation of plantar pressure distribution in obese and non-obese adults. Clin Biomech. 2004;19(10):1055-9. 
determine gait of that individual. The data from various subjects based on walking speed, gender and weight were collected and analyzed.

Thus, the device was made successfully using economically accessible components. At the end of the project, the device is found to be portable, economical, reliable and accessible which can be used in various sectors.

\section{Acknowledgment}

Our project "Design of wireless device to measure foot plantar pressure and gait analysis" required support and guidance from our supervisors, teachers, and family. We find ourselves fortunate for having the opportunity to work on this project under the guidance of supervisors and teachers.

We would like to express our gratitude towards our supervisors Assoc. Prof. Shekhar Khanal and Asst. Prof. Sakar Niraula whose excellent suggestions with positive approach and guidance throughout the project duration have been of great moral support to us. It is a genuine pleasure to express our deepest sense of thanks and gratitude to Dr. Surya Prasad Adhikari, the respected director of CBEAS, whose support encouraged us to conduct this project. Our special thanks go to Asst. Prof. Mishal Pokherel, Er. Menam Pokherel and Er. Prassidha Guragain for their guidance and support throughout the project. We would like to express our gratitude to the College of Biomedical Engineering and Applied Sciences for providing us a platform and a good working environment to transform our ideas into reality.

We would like to thank all the staff members of CBEAS for their support throughout this project. 